\title{
NOTICIAS CIENTÍFICAS Y TÉCNICAS DEL SIGLO XIX RECUPERADAS DEL BOLETÍN OFICIAL DE LA PROVINCIA DE BADAJOZ (ESPAÑA): ANÁLISIS DE REDES
}

\author{
M. ${ }^{a}$ del Rosario Fernández Falero* \\ Facultad de Ciencias de la Documentación y la Comunicación. Universidad de Extremadura. \\ Libertad Sánchez Gil \\ Facultad de Ciencias de la Documentación y la Comunicación. Universidad de Extremadura. \\ Diego Peral Pacheco** \\ Facultad de Ciencias de la Documentación y la Comunicación. Universidad de Extremadura.
}

\begin{abstract}
Resumen: En este trabajo se recuperan y clasifican noticias científicas y técnicas del Boletín Oficial de la Provincia de Badajoz (España), correspondientes al primer semestre del año 1880, con la Nomenclatura Internacional de la $\mathrm{UNESCO}^{1}$ para la Ciencia y la Tecnología, para su posterior automatización. Una vez clasificadas y contabilizadas las noticias, se tabulan los resultados y la representación matricial resultante se estudia mediante el análisis de redes, mostrando las tendencias de la Ciencia y la Tecnología en esta época, así como las relaciones entre las distintas áreas.

En conclusión, podemos determinar que el análisis de la red de relaciones de las noticias científicas y técnicas es un método válido, dentro de las Ciencias Sociales, para el estudio de la información recuperada a través de las diferentes investigaciones historiográficas.

Palabras clave: Cienciometría; Nomenclatura de la UNESCO; Boletín Oficial de la Provincia de Badajoz; siglo XIX.

Title: SCIENTIFIC AND TECHNICAL NEWS OF $19^{\mathrm{TH}}$ CENTURY RETRIEVED FROM THE OFFICIAL GAZZETE OF THE PROVINCE OF BADAJOZ (SPAIN): NETWORK ANALYSIS.

Abstract: In this paper we retrieve and classify scientific and technical news from the Official Gazette of the Province of Badajoz (Spain), of the first semester of 1880. We have classified them with the UNESCO International Nomenclature for Science and Technology. Once the news has been sorted and counted, we have tabulated the results and studied the resulting matrix using network analysis, to show the trends of Science and Technology at this time, and the relationships between different areas.

In conclusion, we can state that the analysis of the network of scientific and technical information is a valid method to study the information retrieved through different historical researches.

Keywords: Information retrieval; scientometrics; UNESCO Nomenclature for the fields of Science and Technology; Official Gazette of the Province of Badajoz; nineteenth century.
\end{abstract}

\section{INTRODUCCIÓN.}

Durante el reinado de Fernando VII ${ }^{2}$ se inicia la publicación del Boletín Oficial de la Provincia de Badajoz mediante la Real Orden de 20 de Abril de 1833 (Pérez, 2000). Se caracteriza por ser una fuente de información de confianza, dado que han sido cotejados los documentos originales y los impresos en otro boletín oficial, La Gaceta de Madrid, con resultados satisfactorios (Romero, 2002), siendo por tanto usada en estudios sobre historia de la ciencia y la técnica. Las gacetas, y por extensión los boletines oficiales, son usados para la recuperación de información de un momento -en este estudio, el siglo XIX- y de un lugar concreto -Badajoz- (Peral, 2007). De hecho se llegó a utilizar como publicación científica: tal es el caso de la medicina homeopática, ya que en 1838 el Doctor Don Pedro Rino y Hurtado "ante la imposibilidad de publicar un nuevo periódico médico, utiliza el Boletín Oficial de la Provincia de Badajoz para animar a los médicos españoles a estudiar la medicina homeopática sin prejuicios" (Fernández Sanz, 1999).

El análisis de redes es un método de trabajo utilizado en Ciencias Sociales para el estudio de las relaciones; en el caso de Biblioteconomía y Documentación es muy utilizado en los análisis y medidas de la actividad científica (Cienciometría); en Historia de la Ciencia se caracteriza por ser un campo de aplicación de los estudios métricos

\footnotetext{
*rferfal@alcazaba.unex.es

**diego@unex.es
}

Recibido: 07/11/2012; 2a versión: 10/01/2014; aceptado: 13/01/2014.

FERNÁNDEZ FALERO, M.R.; SÁNCHEZ GIL, L. y PERAL PACHECO, D. Noticias científicas y técnicas del siglo XIX recuperadas del Boletín Oficial de la Provincia de Badajoz (España): análisis de redes. Anales de Documentación, 2014, vol. 17, $\mathrm{n}^{\circ}$ 1. Disponible en: http://dx.doi.org/10.6018/analesdoc.17.1.162461 
(Araujo, 2002), siendo E. Hulme quién realizó uno de los primeros trabajos, a los que posteriormente se denominó Cienciométricos y que él llamó Bibliografía Estadística (Hulme, 1923). Más recientes son: el trabajo sobre la colaboración, los hábitos de publicación y la difusión de las investigaciones realizadas sobre historia de la ciencia, durante el periodo 1900-2009 en España (Osca-Lluch, 2010), y el de René Sigist y Eric Widmer sobre la formación de los botánicos europeos en el siglo XVIII, donde se realiza un análisis de los subgrupos de botánicos occidentales activos desde 1700 hasta 1830 (Sigrist, 2011). El más actual es el elaborado por Frédéric Vandermoere y Raf Vaderstraeten que establece las redes de comunicaciones entre los estudios de Ciencia y Tecnología y los de historia de la ciencia, determinando que los primeros presentan redes más permeables a otras ciencias; de hecho la Historia de la Ciencia depende en parte de la ciencia y la tecnología para llegar a otras disciplinas (Vandermoere, 2012).

Para la realización de este trabajo se parte de un trabajo previo en el cual se clasificaron las informaciones recuperadas con la Nomenclatura Internacional de la UNESCO para los campos de Ciencia y la Tecnología, creando una base de datos de noticias científicas y técnicas publicadas en el BOPB ${ }^{3}$ en el primer semestre de 1880 , así como un método adecuado para la descripción de las mismas. Posteriormente, se realizó un análisis cuantitativo que mostró cuáles eran los campos científicos y técnicos preponderantes en función del mayor o menor número de noticias censadas. Los resultados mostraron que el campo relativo a la economía era numéricamente el más representativo de la época; seguido de agricultura, etc., lo cual es lógico, dada la época histórica que se analiza, un momento de descentralización del poder administrativo, que a su vez suponía graves problemas recaudatorios. El BOPB daba a conocer, no sólo las propiedades, número de habitantes de cada pueblo, sino también se establecían a través de él, las tasas, los impuestos, así como los edictos y mandatos del Gobierno para el conocimiento de la ciudadanía (Fernández Falero, 2007).

\section{SITUACIÓN HISTÓRICA EN EXTREMADURA.}

En el siglo XIX Extremadura era una región con una densidad poblacional muy baja que fue aumentando paulatinamente sobre todo a partir de la expansión agraria, gracias entre otras cosas a la legislación de colonia agrícola (31.011 Ha. podían acogerse, lo que suponía casi el 10 por 100 del total nacional, siendo Badajoz la provincia extremeña donde más extensión alcanzaban tales colonias) (Zapata, 1996).

La pirámide de población extremeña mostraba un perfil joven, como consecuencia de unas tasas de natalidad y de mortalidad más elevadas que la media nacional. Aunque las causas de mortalidad eran diversas, en buena medida venían a depender de situaciones de desnutrición y pobreza (Sánchez Marroyo, 1997). Una de las causas de mayor mortalidad estaba influida por las condiciones de salubridad; con el fin de mejorar estas condiciones y disminuir, de este modo, la posibilidad de una mayor repercusión de las infecciones se empiezan a llevar a cabo medidas sociosanitarias importantísimas, tales como: creación y mejora de alcantarillado; empedrado de calles, favoreciendo de este modo la eliminación de los residuos de las vías por los laterales, y su limpieza. Se carecía de una canalización de aguas, tanto residuales, como potables, en los núcleos de población, no sólo en los pueblos, sino también en las ciudades; no deberíamos olvidar que hasta hace poco, se escuchaba en muchos lugares de España, la expresión: “¡Agua va!”, que nos avisaba que determinados residuos iban a ser lanzados a la calle, lo que sin lugar a dudas suponía un grave problema sociosanitario; así mismo, fue muy importante la Real Cédula dictada por Carlos III el 3 de Abril de 1787 que obligaba al desplazamiento de los cementerios fuera de los núcleos de población; esta normativa es importantísima y de gran contemporaneidad, pues pone de manifiesto la correlación de epidemias con los enterramientos en las iglesias (Peral, 1991).

Todas estas medidas fueron y son muy importantes, pero su aplicación y puesta en marcha no fue nada sencilla en la provincia de Badajoz. Pues en 1880 Extremadura, en general, es una vasta región con núcleos muy pequeños de población, con unas infraestructuras precarias, y con una red de comunicaciones primitiva (Zapata, 1996), derivando de ahí otros problemas y cuestiones que afectan directamente a la zona, como el alto índice de analfabetismo en la época de nuestro estudio, siendo definida todavía, a principios del siglo XX la situación de la Siberia extremeña, como "un estado de cultura atrasadísimo, imperando el analfabetismo" (Junta, 1924). Para combatir esta lacra, Extremadura sigue, al igual que el resto de España, los dictados de sucesivas medidas legislativas tendentes a paliar tales carencias, habilitando numerosos puestos docentes, que en el caso de la enseñanza primaria son sufragados por cada municipio (Sánchez, 2005).

\section{OBJETIVOS E HIPÓTESIS.}

En la Universidad de Extremadura se han realizado varios trabajos de recuperación de noticias científicas en los boletines oficiales de las provincias de Cáceres y Badajoz, estableciendo el mapa científico de la Ciencia en 
Extremadura (Fernández Falero, 2010), (Pérez, 2000), (Gómez García, 2003). El objetivo de este trabajo es utilizar el análisis de redes para establecer conclusiones válidas que ayuden a revelar las relaciones entre las ciencias, indicando cuál era la que dominaba en la fecha sometida a estudio y que impulsaba el avance científico en la región.

La hipótesis de trabajo es que dado que las ciencias están relacionadas entre sí, no se parte de noticias aisladas, sino de una red científica que utiliza las publicaciones oficiales para comunicarse.

\section{MATERIAL Y MÉTODO.}

Los boletines oficiales de la provincia se crean para que cada capital de provincia distribuya un diario o boletín periódico para dar a conocer todas las órdenes, disposiciones e información oficial procedente de los ayuntamientos de los pueblos o de cualquier otra autoridad competente. Esta norma permitió crear y generalizar los boletines oficiales para todas las provincias.

Posteriormente se asigna a los entonces Jefes Políticos la responsabilidad de la edición de los mismos, siendo los órganos decisorios para la inserción de los correspondientes textos, dado que entre sus atribuciones se encontraba la de agentes estatales de comunicación y control de las provincias y de las autoridades locales, a quienes trasladaban las disposiciones dictadas por el Gobierno, verificando su obligado cumplimiento.

La gestión material del servicio de edición de los boletines oficiales de la provincia pasa a ser responsabilidad de las diputaciones provinciales quienes, además, asumen el coste de la edición (España, 2002).

Se localizan los boletines correspondientes al año en que hemos enmarcado nuestro trabajo (1880) en el Archivo de la Diputación Provincial de Badajoz, ya que posee la colección más completa y en mejor estado del B.O.P.B., encuadernado en colecciones anuales desde 1835 hasta la actualidad (Pérez, 2000), y se procedió a la lectura de cada uno de los ejemplares, efectuando una recopilación de todos los artículos que tuvieran alguna relación con materias científico-tecnológicas de acuerdo con la clasificación internacional de la UNESCO para los campos de Ciencia y Tecnología (Ministerio, 2009), (Fernández Falero, 2003).

Se utiliza la clasificación de la UNESCO porque ésta se adapta perfectamente a la naturaleza de las noticias clasificadas, dado que es una clasificación internacional, muy conocida por los investigadores, y caracterizada porque parte de conceptos muy generales, los campos, y va concretando hasta términos más específicos, por lo que se acomoda muy bien a la naturaleza de las noticias, las cuales pueden ser desde muy generales a muy específicas. Esta Nomenclatura fue propuesta en 1973 y 1974 por las Divisiones de Política Científica y de Estadística de la Ciencia y Tecnología de la UNESCO y finalmente adoptada por la extinta Comisión Asesora de Investigación Científica y Técnica. En 1983 por Resolución de 23 de septiembre de 1983 pasa a ser la clasificación utilizada por el Ministerio de Educación y Ciencia (España, 1983). El Consejo Superior de Investigaciones Científicas ha hecho públicas sucesivas modificaciones por acuerdos de su Junta de Gobierno.

Esta Nomenclatura presenta la posibilidad de clasificar la información en apartados (campos) y subapartados (disciplinas y subdisciplinas) más específicos, dadas las características de este trabajo, estos últimos no se consideran y sólo se tiene en cuenta la clasificación por campos.

“Campo: Se refiere a los apartados más generales, codificados en dos dígitos; comprende varias disciplinas” (Ministerio, 2002).

Las noticias recuperadas se clasifican en distintos campos, llegando a clasificarse en 4 como máximo. Finalmente los resultados ya ordenados se tabulan (Anexo I) y se elabora la matriz de relaciones (Anexo II), considerando que dos campos están relacionados si existe alguna noticia clasificada en ambos campos: por ejemplo, matemáticas presenta 4 noticias clasificadas simultáneamente en pedagogía, es decir que 4 noticias sobre pedagogía se refieren a aspectos relacionados con las matemáticas. La matriz resultante es cuadrada, con los mismos números de filas y columnas, como puede verse en el Anexo II, donde se indica la relación entre los campos con 1 y 0 (cuando hay alguna noticia que haya sido clasificada en ambos campos le corresponde el 1 y cuando nunca se han clasificado noticias en esos campos se pone un 0); para ello se realiza un recuento múltiple y se examina con el programa informático UCINET de análisis de redes (Bogartti, 2002).

Indicadores estudiados siguiendo los trabajos de Rodríguez Molina y Fresvinda Mérida (Rodríguez, 2002) y Robert A. Hanneman y Mark Riddle (Hanneman, 2005):

- Diadas: son las relaciones entre dos campos presentes en el grafo 
- Triadas: son las relaciones entre tres campos presentes en el grafo

- Tamaño de la red: se calcula con la fórmula siguiente, $[\mathrm{N}(\mathrm{N}-1)] / 2$, siendo $\mathrm{N}$ el número de nodos (Friedkin, 1981)

- Densidad media: mide la relación entre los lazos presentes sobre el total de uniones posibles

- Distancia media: muestra las relaciones posibles por el camino más corto

- Centralidad: muestra la posición de los parámetros en la red

- Cercanía: mide la distancia media de cada nodo con respecto al resto de nodos de la red, pero sólo valora los vínculos inmediatos, no las posibilidades indirectas de relaciones en la red

- Núcleo: formado por los nodos más relacionados (Seidman, 1983)

- Subgrupo: son estructuras secundarias de la red cuyo análisis permite obtener información sobre el comportamiento del grafo

\section{RESULTADOS.}

Se han revisado 132 boletines, obteniéndose 1.027 noticias sobre Ciencia y Tecnología durante el primer semestre del año 1880. Se clasifican las noticias como se muestra en el Anexo I, donde se aprecia la relación existente entre los campos y el número de noticias clasificadas en cada uno de ellos; asimismo en dicho Anexo I se pone de manifiesto el solapamiento entre los campos (Fernández Falero, 2005).

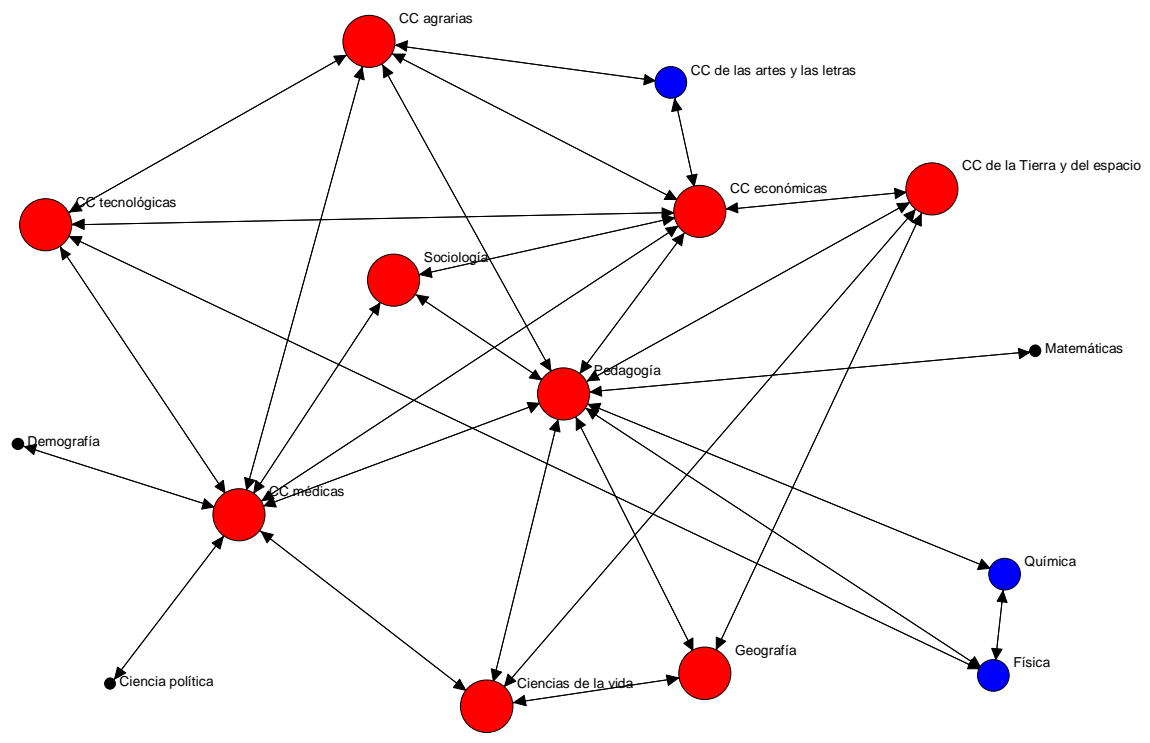

Figura 1. Grafo de las relaciones establecidas entre los campos.

\subsection{Análisis de la red de noticias.}

Una vez establecido el gráfico se analizan las díadas, y se observa que son recíprocas, ya que si una noticia se clasifica en dos campos la relación se establece en ambos sentidos. La mayoría de los campos presenta más de una relación diádica, salvo Demografía, Ciencia Política (relacionadas con CC. Médicas) y Matemáticas (relacionada con Pedagogía). Como se ha mostrado a lo largo del trabajo, la Demografía ${ }^{4}$ está íntimamente ligada a la sanidad, por los problemas epidemiológicos y de salubridad que se habían detectado en diferentes lugares de España; de igual modo, también se ha señalado que el BOPB servía para notificar leyes y normas, por tanto, también se mostraban las diferentes normativas sanitarias que se publicaban, de ahí que no sea extraña la vinculación de la Demografía a las Ciencias Médicas. En el caso de Matemáticas se censan noticias sobre plazas de profesores en Universidades Españolas, de ahí su relación con Pedagogía (hay que recordar que el plan de estudios vigente desde 1850 se modifica el 16 de agosto de 1880 (Cobos, 1998)). 
$\mathrm{Al}$ analizar las triadas se puede ver que CC de las Artes y las Letras es la única que no se relaciona con Pedagogía ni con Medicina, en tanto que el resto se relaciona con las dos o con una de ellas. Se trata de noticias relacionadas con la arquitectura y urbanismo referidas al diseño de la ciudad de Badajoz, resultado de la gestión administrativa; no aparecen noticias culturales clasificables en este campo dado que Badajoz disponía de prensa diaria y por tanto se publicaba en ella (Fernández Fernández, 1989).

Al analizar la red en su totalidad, se observa que no hay nodos aislados, todas las áreas científicas están relacionadas, no hay ninguna rama del conocimiento separada, por tanto las diferentes disciplinas se apoyan las unas en las otras; esto nos muestra una cuestión que se puso con anterioridad de manifiesto: la necesidad de tener una identidad y estudios propios, así como que no hay aún una corriente de especialización claramente definida. No todas las triadas son transitivas o equilibradas, cuando los tres campos tienen todas las relaciones posibles, sino que hay campos que no están conectados; faltan lazos lo que implica una mayor especialización de las noticias en el campo clasificado, y al cuantificar el tamaño máximo de la red, éste indica que existen 105 relaciones posibles. Como bien muestra el análisis de triadas faltan conexiones, lo que se pone de manifiesto al determinar la densidad media, indicando que la probabilidad media de relaciones es de 0,2762 (el 27,62\% de lazos posibles están presentes), lo que demuestra que es una red poco relacionada; es evidente que aunque no hay campos aislados, faltan lazos, debido a que se trata de noticias con un enfoque muy específico y nada generalista.

La distancia media es de 1.905, casi 2, que es la distancia media más corta para que un campo alcance al otro. El diámetro de la red, la distancia más larga entre dos campos, es de 3 y dado que todos los nodos son alcanzables se tiene una red compacta aunque no muy densa. Pese a las pocas relaciones que hay a través del grafo los campos se van relacionando entre sí y creando una red multidisciplinar en ciencia y tecnología.

Vistos los resultados cabía esperar que el campo con más noticias sea el más relacionado, pero no es así; uno de los tres campos con menos relaciones es Demografía, con 36 noticias y el que mayor número de relaciones presenta es pedagogía, con 32 noticias. Este dato tan significativo es producto de la creación en el siglo XIX de los institutos de enseñanza secundaria en el territorio nacional y es en 1845 cuando se establece el primer instituto de enseñanza de la provincia de Badajoz, actualmente denominado Bárbara de Braganza, a petición de la Sociedad Económica de Amigos del País (Tierno, 2008), en la sede del Seminario Conciliar de San Atón fundado en 1644 (Seminario, 2010). La creación de institutos en Extremadura supuso la llegada de profesores que trajeron la Ciencia a la región (Cobos, 2000): teniendo en cuenta esta situación, la Pedagogía es el hilo conductor de la Ciencia en Extremadura y de ahí su posición tan señalada en el análisis de la red.

Respecto a la centralidad (Tabla II) se caracteriza porque es prominente cuando un campo está muy relacionado con todos los campos mientras que estos no tienen relaciones entre sí (Spencer, 2003). Los resultados de UCINET de la centralidad de los nodos del grafo se muestran en la siguiente tabla indicando además la proporción del total de noticias recuperadas en cada campo.

\begin{tabular}{|c|c|c|c|}
\hline Campo & Grado & Proporción & $\begin{array}{c}\mathrm{N}^{\mathrm{o}} \\
\text { noticias }\end{array}$ total $\quad \mathrm{de}$ \\
\hline Pedagogía & 10.000 & 71.429 & 32 \\
\hline CC médicas & 8.000 & 57.143 & 137 \\
\hline CC económicas & 7.000 & 50.000 & 724 \\
\hline $\mathrm{CC}$ agrarias & 5.000 & 35.714 & 197 \\
\hline Ciencias de la vida & 4.000 & 28.571 & 2 \\
\hline CC de la tierra y del espacio & 4.000 & 28.571 & 81 \\
\hline CC tecnológicas & 4.000 & 28.571 & 118 \\
\hline Geografía & 3.000 & 21.429 & 5 \\
\hline Sociología & 3.000 & 21.429 & 67 \\
\hline Física & 3.000 & 21.429 & 14 \\
\hline $\mathrm{CC}$ de las artes y las letras & 2.000 & 14.286 & 15 \\
\hline Química & 2.000 & 14.286 & 1 \\
\hline Demografía & 1.000 & 7.143 & 36 \\
\hline Ciencia política & 1.000 & 7.143 & 2 \\
\hline Matemáticas & 1.000 & 7.143 & 5 \\
\hline
\end{tabular}

Tabla II. Relación entre el grado de centralidad y el número de noticias de cada campo. 


\subsection{A la vista de la Tabla II se hace un estudio por cuartiles.}

En el primer cuartil se encuentra Pedagogía, CC médicas, CC económicas y CC agrarias que se caracterizan porque están muy relacionados con el resto y directamente entre ellos. Respecto a la pedagogía ${ }^{5}$ (Fernández Falero, 2010) en el siglo XIX la ciencia y la tecnología, en conjunto se desarrollaban en el ámbito educativo, de ahí su alto número de relaciones. Aparte del uso por parte de la administración pública de esta publicación oficial, está probada la utilización por los médicos de la época de los boletines oficiales de las provincias españolas como medio de difusión de información médica; a veces actuaba como revista científica, de ahí su situación en esta publicación en relación con otros campos de la ciencia y la tecnología (Fernández Falero, 2005).

En el caso de las CC económicas, el BOPB informa sobre la gestión y administración de la provincia; de ahí el gran número de noticias recuperadas relacionadas con estos aspectos económicos. Por ello su relación con otros campos referentes a impuestos (amillaramientos) y transacciones comerciales, además de tratarse de la necesidad de legislar, controlar y recaudar, controlando la dispersión y el poder de los ayuntamientos y administraciones, desde el Gobierno central. Por esas fechas se está llevando a cabo la actualización de los "registros de la riqueza rústica y pecuaria", mediante la que los ayuntamientos realizaban un reparto de carga fiscal entre los propietarios estableciendo cantidades acordes con su patrimonio (aunque en la realidad había fraudes y ocultación) (Sánchez, 1997). Todas estas circunstancias hacen que en el periodo de tiempo analizado las noticias relacionadas con los sectores agrícola y ganadero, así como económico estén muy presentes.

En el segundo cuartil todos presentan el mismo número de relaciones. En el caso de CC de la tierra y del espacio, donde se clasifican las noticias sobre yacimientos minerales, éstas son numerosas debido a que por esas fechas se empieza a elaborar el mapa geológico y minero de España (Fernández Espinar, 1997); como las noticias sobre minas incluyen el mineral que contienen, ello nos permite observar los conocimientos que se tenían del subsuelo de la provincia, en aquella época.

En cuanto a la cercanía, los más cercanos o centrados son Pedagogía (con un 77,78\% de las relaciones posibles), CC médicas (70\%) CC económicas (66,67\%) y CC agrarias $(60,87 \%)$, que son los que se encuentran más cerca de más nodos que cualquier otro campo, por tanto son los que controlan las relaciones de la red. Resultados esperados por la situación histórica en esas fechas, como ya se ha referido en otras partes de este artículo.

En cuanto a los subgrupos según el análisis k-cores se determina, el núcleo del grafo (en la Figura 1 en color rojo se pueden ver los nodos que forman el núcleo) y al analizarlo muestra 9 campos muy relacionados que forman la base del grafo y que son los que permiten las relaciones entre los mismos; estos son: Pedagogía, CC médicas, CC económicas, CC agrarias, CC de la vida, CC de la tierra y del espacio, CC tecnológicas, Geografía y Sociología, correspondientes a los campos que presentan grados de centralidad que varían desde 10 hasta 3.

Como se ha comentado, la sanidad y la enseñanza son los campos sobre los que se está incidiendo en este momento; por ello forman parte del núcleo. Para el desarrollo de la enseñanza y de la sanidad, es necesario que se apliquen mejoras agrícolas, pues estamos ante una industria primaria, que se sostiene en las capacidades de cultivo, recolecta, y la supervivencia de éstos a las inclemencias del tiempo, así como a las epidemias que sufren las siembras, que hacían perder todo el capital a los agricultores; de igual modo, estamos hablando de una forma de cultivo muy precaria, y en la mayoría de los casos, los beneficios obtenidos de estos cultivos, eran muy pocos y mantenía una situación de pobreza de este colectivo. La necesidad de buscar fuentes de ingreso, llevó a las administraciones a gestionar la demografía desarrollando censos, así como el estudio de la minería.

\section{CONCLUSIONES.}

La Nomenclatura Internacional de la UNESCO para la Ciencia y la Tecnología, además de clasificar noticias, permite también realizar análisis de redes para establecer la existencia de correlaciones entre tales noticias y extraer conclusiones válidas históricamente, como se comprueba en este trabajo.

El número de noticias recuperadas en un campo no es proporcional al número de relaciones de ese campo. Así, Pedagogía y Medicina muestran un mayor número de relaciones, debido fundamentalmente a que en la época del estudio la administración estaba llevando a cabo una importante labor de desarrollo de la sanidad y la enseñanza entre la población. En relación con lo anterior, es curioso destacar que el BOPB no sólo publica noticias legislativas, sino que en ocasiones se utiliza como "revista de divulgación científica". La presencia de las Ciencias agrarias en el primer cuartil del análisis confirma que la economía extremeña es predominantemente agrícola y ganadera. 
El análisis de redes resalta aquellos campos con mayor número de relaciones, que son los que actúan como guías e impulsores del desarrollo científico y técnico del momento en estudio. Puede ayudar como una herramienta verdaderamente objetiva a discriminar resultados aparentes situándolos con su verdadero valor, confirmando la hipótesis del trabajo.

\section{NOTAS}

${ }^{1}$ Sistema de clasificación del conocimiento, creado y desarrollado por la UNESCO, ampliamente usado en la ordenación de proyectos de investigación y de tesis doctorales.

${ }^{2}$ Fernando VII de Borbón (San Lorenzo de El Escorial, 14 de octubre de 1784 - Madrid, 29 de septiembre de 1833), 1lamado el Deseado.

${ }^{3}$ Boletín Oficial de la Provincia de Badajoz.

${ }^{4}$ Los ayuntamientos publican la estadísticas demográfico-sanitarias en el BOPB y en estas fechas (INE, 1888) la Dirección General del Instituto Geográfico y Estadístico publica un censo provisional en 1879 y el definitivo en 1883.

${ }^{5}$ Pedagogía: Ciencia que se ocupa de la educación y la enseñanza (Diccionario de la R.A.E.).

\section{BIBLIOGRAFÍA.}

ARAÚJO RUIZ, J.A.; ARENCIBIA JORGE, R. Informetría, bibliometría y cienciometria: aspectos teóricoprácticos. ACIMED, 2002, vol. 10, nº 4, p. 1-5.

ARDANUY, J.; URBANO, C. y QUINTANA, L. The evolution of recent research on Catalan literature through the production of $\mathrm{PhD}$ theses: a bibliometric and social network analysis. Information Research, 2009, vol. 14, $\mathrm{n}^{\circ} 2, \mathrm{p}$. 1-18 [en línea]. Disponible en: <http://InformationR.net/ir/14-2/paper404.html> [Consulta: 3 de octubre de 2012].

ARROYO, N. et al. Cibermetría estado de la cuestión. En FESABID. $9^{a}$ Jornadas españolas de documentación, Madrid: FESABID, 2005, p. 273-290.

BORDER, A.; KUMAR, R.; MAGHOUL, F.; RAGHAVAN, P.; RAJAGOPALAN, S.; STATA, R.; TOMKINS, A. y WIENER, J. Graph structure in the web. Computer Networks, 2000, vol. 33, p. 309-320.

BORGATTI, S.P., EVERETT, M.G. y FREEMAN, L.C. Ucinet for Windows: Software for Social Network Analysis. Harvard: Analytic Technologies, 2002.

COBOS BUENO, J.M.; PERAL PACHECO, D. y VAQUERO MARTÍNEZ, J.M. Ciencia en Extremadura en el tránsito del siglo XIX al XX. Revista de Estudios Extremeños, 1998, vol. 54, nº 1, p. 427-470.

COBOS BUENO, J.M. y VAQUERO MARTÍNEZ, J.M. Materiales para una Historia de la Ciencia en Extremadura. Cáceres: Servicio de Publicaciones (UEX), 2000.

ESPAÑA. Ley 5/2002, de 4 de abril, reguladora de los Boletines Oficiales de las Provincias. Boletín Oficial del Estado, 5 de abril de 2002, núm. 82, p. 12988.

ESPAÑA, Resolución de 23 de septiembre de 1983 por la que se publica la relación total de campos, disciplinas y subdisciplinas de especialización científica y tecnológica en los que desarrollan su actividad los Institutos, Centros e Investigadores dependientes del mismo. Boletín Oficial del Estado, 14 de octubre de 1983, núm. 246, p. 27855-27868.

FERNÁNDEZ-ESPINAR, L.C. Derecho de minas en España (1825-1996). Granada: Editorial Comares, 1997.

FERNÁNDEZ FALERO, M.R.; HERRERA MORILLAS J.L. y PERAL PACHECO, D. Las noticias sanitarias en el Boletín Oficial de la Provincia de Badajoz. Revista de Estudios Extremeños, 2003, vol. 59, no 1, p. 79-91.

FERNÁNDEZ FALERO, M.R. Aplicación de la documentación a la recuperación de noticias del Boletín Oficial de la Provincia de Badajoz. Badajoz: Diputación Provincial de Badajoz (imp.), 2005.

FERNÁNDEZ FALERO, M.R. y PERAL PACHECO, D. Las publicaciones oficiales como medio de comunicación de la información científica y técnica a través del tiempo. Razón y Palabra, 2007, vol 12, nº 56, p. 1-11 [en línea]. Disponible en: <http://www.razonypalabra.org.mx/anteriores/n56/fernandezperal.html $>$ [Consulta: $1 \mathrm{de}$ octubre de 2012].

FERNÁNDEZ FALERO, M.R. La Documentación Científica en el Boletín Oficial de la Provincia de Badajoz: 1880. Cáceres: Servicio de Publicaciones, Universidad de Extremadura, 2010.

FERNÁNDEZ FERNÁNDEZ, P.V. Prensa promasónica y antimasónica en el Badajoz de fines del siglo XIX. En Ferrer Benimeli, J.A. (ed) Masonería, política y sociedad. Centro de Estudios Históricos de la Masonería Española, 1989, p. 281-290.

FERNÁNDEZ SANZ, J.J. La prensa homeopática española en el siglo XIX’. Madrid: Fundación Instituto Homeopático y Hospital de San José Federación Española de Médicos Homeópatas, 1999.

FRIEDKIN N.E. The development of structure in random network: an análisis of the effects of increasing network density on five measures of structure. Social Network, 1981, vol. 3, p. 41-52.

GÓMEZ GARCIA, P.; GONZÁLEZ GALÁN, V. y GONZÁLEZ CASTILLO, P. Noticias sanitarias recogidas en el Boletín Oficial de la Provincia de Cáceres (1833-1840). Revista de Estudios Extremeños, 2003, vol. 59, nº 1, p. $119-$ 144. 
HANNEMAN, R. y RIDDLE, M. Introduction to social network methods. Riverside, CA: University of California, Riverside. 2005. Disponible en: <http://faculty.ucr.edu/ hanneman/> [Consulta: 18 de septiembre de 2013].

HULME, E.W. Statistical bibliography in relation to the growth of modern civilization. London: Grafton, 1923 [en línea]. Disponible en: <http://archive.org/stream/statisticalbibli00hulmuoft\#page/n5/mode/2up $>$ [Consulta: 3 de octubre de 2012].

INE. Reseña Geográfica y Estadística de España, 1888 [en línea]. Disponible en: $<$ http://www.ine.es/inebaseweb/treeNavigation.do?tn=192688> [Consulta: 29 de octubre de 2012].

Junta Central de Colonización y Repoblación Interior. Sucinta información de las colonias agrícolas instaladas y en periodo de establecimiento o estudio. Madrid: Gráficas reunidas, 1924.

LEYDESDORFF, L. y VAUGHAN, L. Co-occurrence Matrices and their Applications in Information Science: Extending ACA to the Web Environment. Journal of the American Society for Information Science and Technology, 2006, vol. 57, n ${ }^{\circ} 12$, p. 1616-1628.

Ministerio de Ciencia e Innovación. Nomenclatura Internacional de la UNESCO para los campos de Ciencia y Tecnología, $2009 \quad$ [en línea]. en: $<$ http://www.micinn.es/portal/site/MICINN/menuitem.8ce192e94ba842bea3bc811001432ea0/?vgnextoid=f0918957a2 2d0210VgnVCM1000001034e20aRCRD\&vgnextchannel=9fc8603368ce2210VgnVCM1000001d04140aRCRD\#idPr eguntas $>$ [Consulta: 12 de octubre de 2012].

OSCA-LLUCH, J. Aplicación del análisis de redes al estudio de la investigación española de historia de la ciencia. Revista redes, 2010, vol. 19, p. 122-143.

PERAL PACHECO, D. El cólera y los cementerios en el siglo XIX. Norba: Revista de Historia, 1991-1992, $\mathrm{n}^{\circ} 11-$ 12, p. 271-280.

PERAL PACHECO, D. y PÉREZ TORRALBA, T. Las enfermedades epidémicas en el Boletín Oficial de la Provincia de Badajoz en el siglo XIX (1833-1873). Norba: Revista de Historia, 2007, vol. 20, p 143-160.

PÉREZ TORRALBA, T. La Documentación Sanitaria en el Boletín Oficial de Badajoz en el siglo XIX: 18331850. Badajoz: Universidad de Extremadura, 2000. (Inédita).

RODRÍGUEZ, J.A. Análisis estructural y de redes. Madrid: Centro de Investigaciones Sociológicas (CIS), 2005.

RODRÍGUEZ, J.A. y MÉRIDA DE PEDRAZA, F. Guía práctica de redes sociales con Ucinet 6 . Universidad de Barcelona. Departamento de Sociología y Análisis de las Organizaciones, 2002.

ROMERO TALlAFIGO, M. Historia del documento en la edad contemporánea: la comunicación y la representación del poder central de la nación. Carmona: S\&C Ediciones, 2002.

SÁNCHEZ MARROYO, F. (ed.). Extremadura, la historia. Badajoz: HOY, diario de Extremadura, 1997.

SÁNCHEZ PASCUA, F. Los orígenes de la educación reglada obligatoria en Extremadura. Revista de Estudios Extremeños, 2005, vol. 61, nº 3, p. 883-920.

SANZ MENÉNDEZ, L. Análisis de redes sociales: o como representar las estructuras sociales subyacentes. Apuntes de Ciencia y Tecnología, 2003, n 7, p. 20-29.

Seminario Metropolitano de San Atón. Historia [en línea]. Disponible en: $<$ http://seminario.meridabadajoz.es/index.php?option=com_content\&task=view\&id=118\&Itemid=102> [Consulta: 7 de octubre de 2012].

SEIDMAN, S.B. Network structure and minimum degree. Social Network, 1983, nº 5, p. 269-287.

SIGRIST, R. y WIDMER, E.D. Vínculos de formación y transmisión del conocimiento en la botánica del siglo XVIII: Análisis de redes sociales. Revista Redes, 2011, vol. 21, p. 301-346.

SPENCER, J.W. Global gatekeeping, representation and network structure: a longitudinal analysis of regional and global knowledge-diffusion networks. Journal of international business studies, 2003, vol. 34, p. 428-442.

TIERNO VELASCO, R. IES Bárbara de Braganza Badajoz. CEE Participación Educativa, noviembre 2008 , vol. 9, p. 120-130.

VANDERMOERE, F. y VANDESTRAETEN, R. Disciplinary Networks and Bounding: Scientific Communication Between Science and Technology Studies and the History of Science. Minerva, 2012, vol. 50, p. 451470 .

VILLANUEVA SERRANO, S.J. et al. Análisis de la red de colaboración científica sobre tabaquismo en centros sanitarios españoles a través del Science Citation Index (1999-2003). Archivos de Bronconeumología, 2007, vol. 43, $\mathrm{n}^{\mathrm{o}} 7$, p. 378-385

ZAPATA BLANCO, S. (ed.). La industria de una región no industrializada: Extremadura, 1750-1990. Cáceres: Universidad de Extremadura, Servicio de Publicaciones, 1996. 
Anexo I. Tabla de resultados: clasificación por campos de las noticias recuperadas.

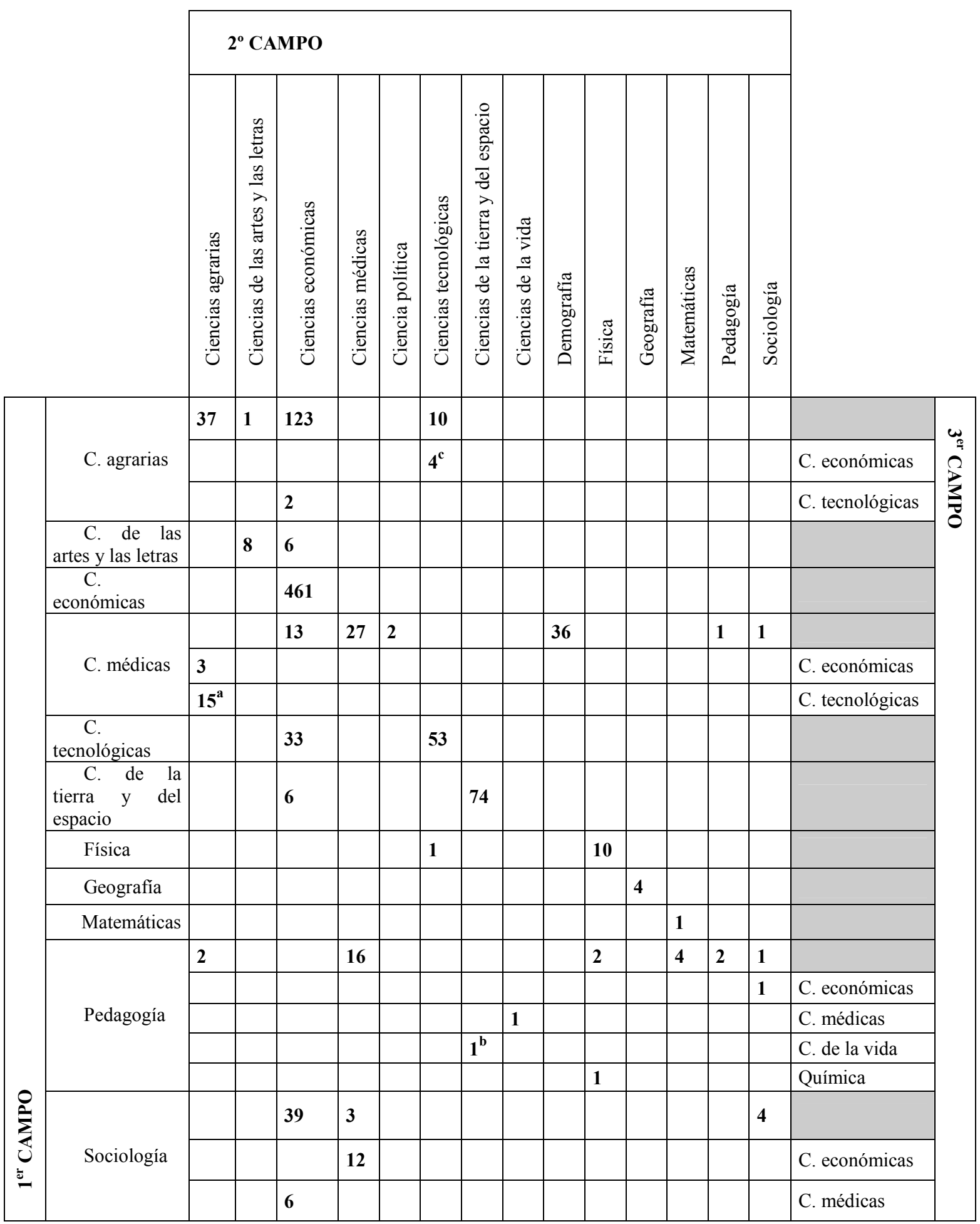

Tabla I. Distribución de las noticias en varios campos. (Fernández 2010).

a) = Se relacionan también con un cuarto campo: CIENCIAS ECONÓMICAS

b) $=$ Se relaciona también con el campo GEOGRAFÍA

c) = Una de ellas se relaciona también con CIENCIAS MÉDICAS 
Anexo II. Matriz de relaciones.

\begin{tabular}{|c|c|c|c|c|c|c|c|c|c|c|c|c|c|c|c|}
\hline & $\begin{array}{c}\mathrm{CC} \\
\text { agrarias }\end{array}$ & \begin{tabular}{|c|} 
CC de \\
las artes \\
y las \\
letras \\
\end{tabular} & $\begin{array}{l}\mathrm{CC} \\
\text { econó } \\
\text { micas }\end{array}$ & $\begin{array}{c}\mathrm{CC} \\
\text { médicas }\end{array}$ & $\begin{array}{l}\text { Ciencia } \\
\text { política }\end{array}$ & $\begin{array}{c}\mathrm{CC} \\
\text { tecnológi } \\
\text { cas }\end{array}$ & $\begin{array}{c}\text { CC de la } \\
\text { tierra y } \\
\text { del } \\
\text { espacio } \\
\end{array}$ & $\begin{array}{l}\text { CC de } \\
\text { la vida }\end{array}$ & Demografía & Física & Geografía & Matemáticas & Pedagogía & Química & Sociología \\
\hline $\mathrm{CC}$ agrarias & 1 & 1 & 1 & 1 & 0 & 1 & 0 & 0 & 0 & 0 & 0 & 0 & 1 & 0 & 0 \\
\hline \begin{tabular}{|l|}
$\mathrm{CC}$ de las artes y \\
las letras
\end{tabular} & 1 & 1 & 1 & 0 & 0 & 0 & 0 & 0 & 0 & 0 & 0 & 0 & 0 & 0 & 0 \\
\hline CC económicas & 1 & 1 & 1 & 1 & 0 & 1 & 1 & 0 & 0 & 0 & 0 & 0 & 1 & 0 & 1 \\
\hline CC médicas & 1 & 0 & 1 & 1 & 1 & 1 & 0 & 1 & 1 & 0 & 0 & 0 & 1 & 0 & 1 \\
\hline Ciencia política & 0 & 0 & 0 & 1 & 0 & 0 & 0 & 0 & 0 & 0 & 0 & 0 & 0 & 0 & 0 \\
\hline CC tecnológicas & 1 & 0 & 1 & 1 & 0 & 1 & 0 & 0 & 0 & 1 & 0 & 0 & 0 & 0 & 0 \\
\hline $\begin{array}{l}\text { CC de la Tierra y } \\
\text { del espacio }\end{array}$ & 0 & 0 & 1 & 0 & 0 & 0 & 1 & 1 & 0 & 0 & 1 & 0 & 1 & 0 & 0 \\
\hline $\begin{array}{|lll|}\begin{array}{l}\text { Ciencias de la } \\
\text { vida }\end{array} & & \\
\end{array}$ & 0 & 0 & 0 & 1 & 0 & 0 & 1 & 0 & 0 & 0 & 1 & 0 & 1 & 0 & 0 \\
\hline Demografía & 0 & 0 & 0 & 1 & 0 & 0 & 0 & 0 & 0 & 0 & 0 & 0 & 0 & 0 & 0 \\
\hline Física & 0 & 0 & 0 & 0 & 0 & 1 & 0 & 0 & 0 & 1 & 0 & 0 & 1 & 1 & 0 \\
\hline Geografía & 0 & 0 & 0 & 0 & 0 & 0 & 1 & 1 & 0 & 0 & 1 & 0 & 1 & 0 & 0 \\
\hline Matemáticas & 0 & 0 & 0 & 0 & 0 & 0 & 0 & 0 & 0 & 0 & 0 & 1 & 1 & 0 & 0 \\
\hline Pedagogía & 1 & 0 & 1 & 1 & 0 & 0 & 1 & 1 & 0 & 1 & 1 & 1 & 1 & 1 & 1 \\
\hline Química & 0 & 0 & 0 & 0 & 0 & 0 & 0 & 0 & 0 & 1 & 0 & 0 & 1 & 0 & 0 \\
\hline Sociología & 0 & 0 & 1 & 1 & 0 & 0 & 0 & 0 & 0 & 0 & 0 & 0 & 1 & 0 & 1 \\
\hline
\end{tabular}

